\title{
TRANSFER ENTROPY ESTIMATION SUPPLEMENTS TIME-DOMAIN BEAT-TO-BEAT BAROREFLEX SENSITIVITY INDEX UNDER POSTURAL STRESS
}

\author{
B S Saini ${ }^{1}$, A Singh ${ }^{2}$, D Singh ${ }^{3}$ \\ ${ }^{1,3}$ Associate Professor, ${ }^{2}$ Research Scholar, Department of ECE, National Institute of Technology, Punjab, India, \\ sainibss@gmail.com, apsingh.lpu@gmail.com,drdilbag@gmail.com
}

\begin{abstract}
Baroreflex mechanism plays a vital role in cardiovascular regulation by contributing in sympathetic-vagal imbalance triggered by baroreceptor reflex. This study estimates BRS (Baroreflex Sensitivity) index from beat-to-beat systolic blood pressure (SBP) and RR interval (RRi) series using time-domain windowed cross-correlation method. This index is supplemented by an information domain technique called Transfer Entropy (TE) is applied to decipher the directional coupling between SBP and RRi series. The study is performed on EUROBAVAR data. The results show that BRS index calculated is large $(1.157 \pm 0.533)$ in supine position which supplemented by TE $(S B P-R R)$ of $(0.129 \pm 0.09)$ as compared to standing position for which BRS index is $(0.866 \pm 0.472)$ and a TE $(S B P-R R)$ of $(0.04 \pm 0.03)$ for EUROBAVAR data with a p-value $<0.05$. Also, it is found that both the TE indices i.e. from $S B P$ to $R R$ and from RR to SBP show correlated results with the time domain BRS index in both supine and standing positions. Further, the cases with possible BRS failure are having very small values of BRS index, which are not assigned any value by prevalent methods. This shift is absent in BRS failure patients which is supplanted by TE (RR-SBP) sharing more information bits than TE (SBP-RR). Further, it is observed that patients having baroreflex impairment show reversal of information flow which is indicated by a larger TE (RR$S B P)$ index as compare to TE (SBP-RR) index. Time domain BRS index lacks directional information which is provided by TE and hence this supplemental information gives better interpretation of BRS index especially in patients having suppressed baroreflex.
\end{abstract}

Index Terms: Baroreflex sensitivity, Directional coupling, Cross-correlation, and Transfer entropy

\section{INTRODUCTION}

Baroreflex modulates both cardiac parasympathetic and sympathetic outflow to the sinus node in the heart. Quantification of this baroreflex control can be done by an index known as Baroreflex Sensitivity (BRS) which depends on the rate of change of heart rate corresponding to rate of change of systolic pressure [1]. Cardiac baroreflex sensitivity index is known to have been influenced by certain cardiac autonomic function tests viz. supine-to-standing test, Valsalva manoeuvre, deep breathing, cold pressor test and handgrip test $[2,3]$.

In the Task Force paper [4], it has been quoted that unlike heart rate variability (HRV), BRS index is able to distinguish between post myocardial infarction cardiac arrest (MI-CA) survivors from other post MI patients. BRS index is also able to assess autonomic dysfunction of diabetic patients [5]. Over the last two decades, BRS index has emerged as a great prognostic indicator of wide range of cardiovascular diseases [6,7]. Both invasive and non-invasive methods have been developed over a period of time to analyze baroreflex sensitivity. The invasive methods use external stimulus such as drugs to vary the blood pressure thereby activating the baroreflex. Recent methods use non-invasive means of evaluating BRS and are based on spontaneous beat-to-beat measurement of both blood pressure and heart rate. Sequence method and cross correlation method are among the popular time domain methods. Frequency domain methods include parametric and non-parametric spectral estimation to estimate low frequency (LF) and high frequency (HF) gain [8]. Some studies have also taken into account the phase or time-delays between beat-to-beat systolic pressure and RR intervals series to estimate BRS [9]. Directional coupling information needs to be quantified to provide a more complete estimation of BRS index which cannot be calculated using linear time domain index. Some earlier studies [9] have utilized mutual information and time delayed mutual information to calculate amount information exchanged between the two signals which are generated by a same system. In this paper, a BRS index obtained using beat-to-beat cross-correlation analysis of SBP and $\mathrm{RRi}$ series supplemented by calculating the directional coupling index using information theory based Transfer Entropy method is presented. 


\section{DATA SAMPLES AND PRE-PROCESSING}

21 subjects from EUROBAVAR dataset (4 males and 17 females) aged 20 to 68 years $(38 \pm 14.7$, mean \pm SD) are taken for BRS estimation. In the EUROBAVAR dataset there are 4 healthy volunteers, 8 outpatients, 3 hypertension patients, 2 hypercholesterolemia patients, 1 diabetic without neuropathy, 1 diabetic with neuropathy (BRS failure), 1 heart transplant patient (BRS failure) and 1 pregnant woman.

\subsection{Data Pre-processing}

Systolic blood pressure (SBP) values and R-peaks are detected using an algorithm based on empirical mode decomposition (EMD). The EMD method decomposes the ECG signal and Blood Pressure (BP) signal into a set of oscillatory components called intrinsic mode functions (IMFs). This is a data driven method unlike wavelet transformation which decomposes the input signal based on a pre-defined kernel [11]. Ectopic beats were manually removed from the EUROBAVAR as well as from the recorded data. From the detected peaks of both ECG and BP signal, time series of RR intervals (RRi) and SBP values are formed. The SBP and RRi series for $n$ number of samples are normalized by subtracting and then dividing by the mean.

For TE implementation an increase in RR interval and SBP value is taken as "1" and a decrease in subsequent values is taken as " 0 ". In this way a series of binary symbols is generated which is used to calculate the transfer entropy from SBP to RR and from RR to SBP.

\section{METHODS}

Both time domain and information theory based methods are applied to quantify the mutual coupling between SBP and RRi time series. Time domain method is a linear technique which provides BRS index but does not provide bi-directional interaction between SBP and RRi series whereas information theory based TE method quantify directional coupling between the two series under consideration. A detailed explanation these techniques are as follows.

\subsection{Time domain cross-correlation method}

Beat-to-beat SBP and RRI series are considered for each subject. Beat events are synchronized according to RR intervals. A 10 beat sliding window is used to find the correlation between each SBP value and RR interval. To start with, SBP values corresponding to first 10 beats were considered and their cross correlation with first 10 RRI values is performed using the following equation [1]:

$$
\begin{gathered}
\rho= \\
N \sum_{i=1}^{N} R R I_{n}(i) \cdot S B P_{n}(i)-\sum_{i=1}^{N} R R I_{n}(i) \sum_{i=1}^{N} S B P_{n}(i) \\
\hline
\end{gathered}
$$

$$
\sqrt{\left\{N \sum_{i=1}^{N}\left(R R I_{n}(i)\right)^{2}\right\}-\left\{\left(\sum R R I_{n}(i)\right)^{2}\right\}} \sqrt{\left\{N \sum_{i=1}^{N}\left(S B P_{n}(i)\right)^{2}\right\}-\left\{\left(\sum S B P_{n}(i)\right)^{2}\right\}}
$$

This first calculation of cross-correlation corresponds to delay of zero beat. The next calculation is carried out on the same 10 SBP values and the interval values shifted by 1 beat. $\left(2^{\text {nd }}\right.$ to $11^{\text {th }}$ beat). The window is shifted by one beat sample till 15th RRI value and the cross-correlation is estimated for the same 10 SBP values and the shifted RRI values at each window slide. The maximum of the cross-correlation value is selected out of these 6 cross-correlation values. Regression slope is estimated between the SBP and RRI values corresponding to the maximum cross-correlation value according to the following equation [1]:

$$
\mathrm{r}=\frac{N \sum_{i=1}^{N} R R I_{n}(i) \cdot S B P_{n}(i)-\sum_{i=1}^{N} R R I_{n}(i) \sum_{i=1}^{N} S B P_{n}(i)}{\sqrt{\left\{N \sum_{i=1}^{N}\left(R R I_{n}(i)\right)^{2}\right\}-\left\{\left(\sum R R I_{n}(i)\right)^{2}\right\}}}
$$

This regression slope is taken as BRS index if its value is positive otherwise the corresponding segment is not counted towards estimation of BRS. The average regression slope is calculated and taken as an index of BRS. SBP and RRI values are also influenced by factors other than BRS. To account for this, the accepted regression slope is divided by the correlation coefficient to get an index called non-BRS index.

\subsection{Information theory based Transfer Entropy}

\section{Estimation}

TE has been established as a powerful tool for detecting the transfer of information between two joint processes [11]. TE has a solid foundation in the information theory and it naturally incorporates directional and dynamical information as it is different when computed over two causal directions [11]. TE from a process $\mathrm{j}$ to a process $\mathrm{i}$ is given by the following equations:

$$
\begin{aligned}
& T_{j \rightarrow i}=\sum_{x_{n+1}, x_{n}, y_{n}} p\left(x_{n+1}, x_{n}, y_{n}\right) \log \frac{p\left(x_{n+1}, x_{n}, y_{n}\right) \cdot p\left(x_{n}\right)}{p\left(x_{n}, y_{n}\right) \cdot p\left(x_{n+1}, x_{n}\right)} \\
& T_{i \rightarrow j}=\sum_{y_{n+1}, x_{n}, y_{n}} p\left(y_{n+1}, x_{n}, y_{n}\right) \log \frac{p\left(y_{n+1}, x_{n}, y_{n}\right) \cdot p\left(y_{n}\right)}{p\left(x_{n}, y_{n}\right) \cdot p\left(y_{n+1}, y_{n}\right)}
\end{aligned}
$$

Where $\mathrm{x}$ and $\mathrm{y}$ represents symbolic RRi and SBP series respectively. $\mathrm{p}(\mathrm{x}), \mathrm{p}(\mathrm{y})$ are the individual probabilities and $p(x, y)$ and $p(y, x)$ are the joint probabilities.

\section{RESULTS AND DISCUSSIONS}

\subsection{Cross-correlation BRS index (xBRS)}

This method provides BRS estimates for all subjects of EUROBAVAR dataset and laboratory recorded data. Table 1 provides the mean and standard deviation of the BRS estimates. It is evident that supine BRS values are greater than 
standing BRS values. This is true for EUROBAVAR as well as data recorded under laboratory conditions.

\subsection{Transfer Entropy Index}

Table 1 also shows the TE index calculated from SBP to RR and vice-versa. These two indices convey the directional coupling information between the two time series.

Table-1: BRS index and TE index of healthy subjects

\begin{tabular}{|c|c|c|c|c|}
\hline \multicolumn{2}{|c|}{ Position } & \multicolumn{3}{|c|}{ Coupling Indices (Healthy) } \\
\hline \multirow{3}{*}{ 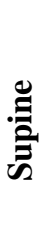 } & & $\begin{array}{l}\text { Time-domain } \\
x \text {-BRS index }\end{array}$ & $\begin{array}{l}\text { TE index } \\
(S B P-R R)\end{array}$ & $\begin{array}{c}\text { TE index } \\
(R R-S B P)\end{array}$ \\
\hline & Mean & 1.157 & 0.13 & 0.11 \\
\hline & SD & 0.533 & 0.09 & 0.09 \\
\hline \multirow{2}{*}{ } & Mean & 0.866 & 0.05 & 0.04 \\
\hline & SD & 0.472 & 0.03 & 0.03 \\
\hline
\end{tabular}

Table 2 provides the BRS index for baro-reflex impaired patients B005 and B010 in both positions. The low values of BRS index confirm this impairment. Directional coupling which is indicated by the two transfer entropy measures also show a certain decrease in coupling as indicated by the decreased TE indices from supine to standing position in healthy subjects.

Table-2: BRS index and TE index of patients with depressed BRS

\begin{tabular}{|c|c|c|c|c|}
\hline \multicolumn{2}{|c|}{ Position } & \multicolumn{3}{|c|}{$\begin{array}{c}\text { Coupling Indices (Patients with depressed } \\
\text { BRS) }\end{array}$} \\
\hline \multirow{3}{*}{ מ゙ } & & $\begin{array}{l}\text { Time-domain } \\
x-B R S \text { index }\end{array}$ & $\begin{array}{l}\text { TE index } \\
(S B P-R R)\end{array}$ & $\begin{array}{l}\text { TE index } \\
(R R-S B P)\end{array}$ \\
\hline & Mean & 0.21 & 0.017 & 0.073 \\
\hline & SD & 0.02 & 0.01 & 0.03 \\
\hline \multirow{2}{*}{ : } & Mean & 0.13 & 0.006 & 0.008 \\
\hline & SD & 0.19 & 0.13 & 0.01 \\
\hline
\end{tabular}

The results do indicate a correlation of directional coupling indices with the time domain $x$-BRS index obtained from windowed cross-correlation method for both supine and standing positions.

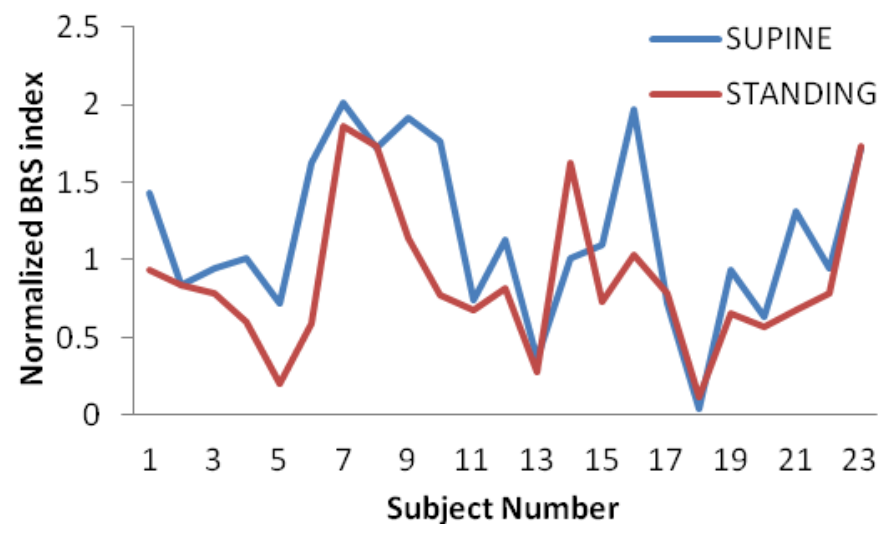

Chart -1: BRS index of all the subjects

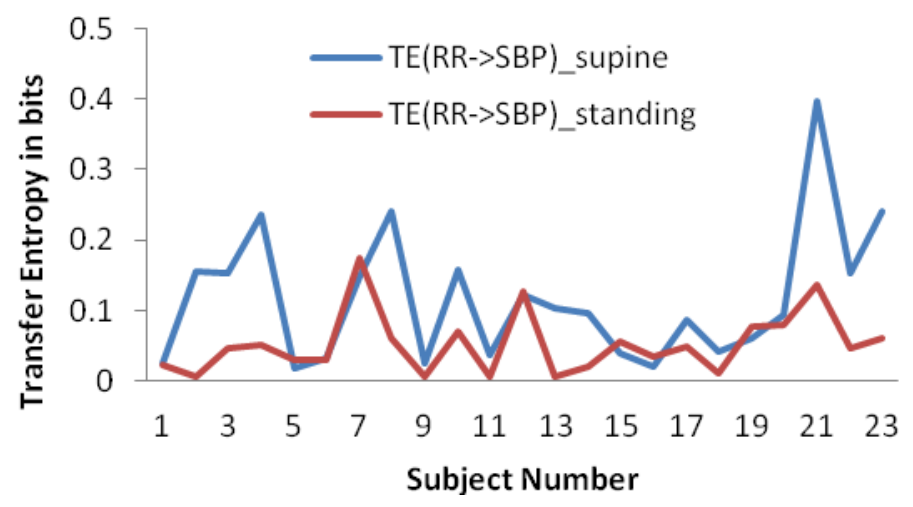

Chart -2: TE (RR-SBP) for all subjects

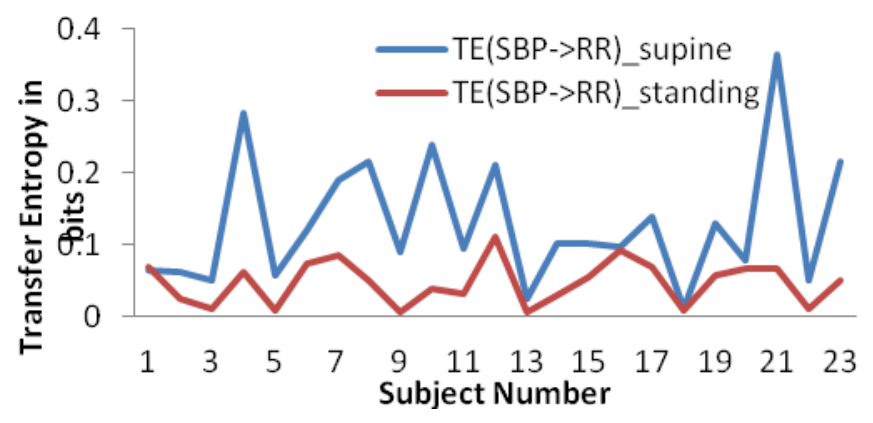

Chart -3: TE (SBP-RR) for all subjects

Chart 1,2 and 3 show that there is a decrease in BRS as well as the coupling indices from supine to standing in most of the subjects. Since the time domain x-BRS index does not provide any directional coupling information, the transfer entropy indices supplement this information. It is worth noting that for healthy subjects in which BRS is intact the TE index from SBP to RR is greater than that of RR to SBP which shows the actual baroreflex action i.e change in SBP leads to change in RR interval. This reverses in case of patients with depressed BRS. 


\subsection{Significance of difference between lying and standing positions}

The cross correlation BRS index and TE indices calculated by above methods differ significantly for both lying and standing positions and was evaluated using chi-square test with a pvalue of 0.004 .

The results also showed significant differences for delay parameter with a p-value less than 0.05. The precision in standing as well as lying position was almost same. Though the magnitudes of pressure values as well as the interval time change with a change in position the sensitivity of BRS index and TE estimation remained almost same

\section{CONCLUSIONS}

Time domain BRS index gives incomplete information and lacks interpretation in diverse patho-physiological cases since it lacks directional coupling information and its linear nature is unable to track non-linear and non-stationary fluctuations that normally occur in complex physiological signals. Transfer entropy index provides directional coupling information between SBP and RR intervals and hence supplements the BRS index obtained from traditional time domain method. The results clearly show that there is a tendency to reverse the direction of information flow in case of conditions such as heart transplantation and diabetic neuropathic conditions in which depressed BRS is recorded. Future work should concentrate on more detailed analysis based on the techniques adopted from information theory.

\section{ACKNOWLEDGEMENTS}

The authors are thankful to the Department of Electronics and Communication Engineering and Department of Instrumentation and Control for providing the necessary laboratory support to carry out a research work.

\section{REFERENCES:}

[1] B. E. Westerhof, J. Gisolf, W. J. Stok, K. H. Wesseling, and J. M. Karemaker, "Time-domain cross-correlation baroreflex sensitivity: Performance on the EUROBAVAR data set”, Journal of Hypertension, vol. 22, pp.1371-1380, 2004.

[2] J. D. Lefrandt, K. Hoogenberg, A. M. Van Roon, R. P. Dullaart, R. O. Gans and A. J. Smit, "Baroreflex sensitivity is depressed in microalbuminuric Type I diabetic patients at rest and during sympathetic manoeuvres", Journal of Diabetologia, vol. 42, pp. 1345-1349, 1999.

[3] D. J. Ewing, C. N. Martyn, R. J. Young and B. F. Clarke, "The value of cardiovascular autonomic function tests: 10 years experience in diabetes", Journal of Diabetes Care, vol. 8, pp. 491-498, 1985.
[4] Task Force of the European Society of Cardiology and the North American Society of Pacing and Electrophysiology, "Heart rate variability-standards of measurement, physiological interpretation and clinical use", European Heart Journal, vol. 17, pp. 354-38, 1996.

[5] V. Spallone, and G. Menzinger, "Diagnosis of cardiovascular autonomic neuropathy in diabetes", Journal of Diabetes, vol. 46, S67-S76, 1997.

[6] M. T. La Rovere, J. T. Bigger Jr., F. I. Marcus, A. Mortara and P. J. Schwartz, "Baroreflex sensitivity and heart-rate variability in prediction of total cardiac mortality after myocardial infarction", Lancet, vol. 351, pp. 478-484, 1998.

[7] D. Laude, J.-Luc Elghozi, A. Girard, E. Bellard, M. Bouhaddi, P. Castiglioni, C. Cerutti, A. Cividjian, M. Di Rienzo, J. O. Fortrat, B. Janssen, J. M. Karemaker, G. Lefthériotis, G. Parati, P. B. Persson, A. Porta, L. Quintin, J. Regnard, H. Rüdiger and H. M. Stauss, "Comparison of various techniques used to estimate spontaneous baroreflex sensitivity (the EuroBaVar study)," Am. J. Physiol., Regulatory, Integrative and Comparative Physiology, vol. 286, pp. R226-R231, 2004.

[8] R. H. Clayton, A. J. Bowman, G. A. Ford and A. Murray, "Measurement of baroreflex gain from heart rate and blood pressure spectra: A comparison of spectral estimation techniques", Physiological Measurement, vol. 16, pp 131, 1995.

[9] S. Khan, S. Bandyopadhyay, A.R. Ganguly, S. Saigal, D.J. Erickson, V. Protopopescu, and G. Ostrouchov, "Relative performance of mutual information estimation methods for quantifying the dependence among short and noisy data," Physical Review E, vol. 76, no. 2, pp. 1-15, 2007

[10] H. Li, S. Kwong, L. Yang, D. Huang and D. Xiao, "Hilbert-Huang Transform for Analysis of Heart Rate Variability in Cardiac Health", IEEE/ACM Transactions on Computational Biology and Bioinformatics, vol. 8, pp. 1557-1567, 2011.

[11] J. Lee, S. Nemati, I. Silva, B.A. Edwards, J.P. Butler, and A. Malhotra, "Transfer entropy estimation and directional coupling change detection in biomedical time series," BioMedical Engineering Online, vol. 11, no. 19, pp. 1-17, 2012.

\section{BIOGRAPHIES:}

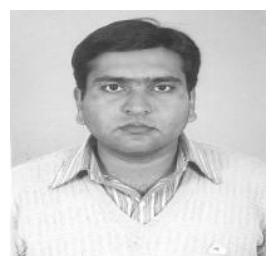

Barjinder S. Saini was born in Jalandhar, India, in 1970. He received his B.Tech and M.Tech. degrees in Electronics \& Communication Engineering in 1994 and 1996 respectively. He then obtained his $\mathrm{PhD}$ degree in Engineering on "Signal Processing of Heart Rate Variability" in 2009 from Dr. B. R. Ambedkar National Institute of Technology, Jalandhar. He is working as Associate Professor 
in Electronics \& Communication Engineering Department at NIT Jalandhar since 1997. He supervised more than 20 M.Tech dissertations and presently guiding $06 \mathrm{Ph}$. D research scholars. He has published more than 20 research papers in internationally reputed Journals and Conference proceedings. $\mathrm{He}$ has accomplished research projects from government departments. He has also conducted several courses, workshops and international conferences for the benefit of faculty and field engineers. His areas of interest are Medical Image Processing, Digital Signal Processing and Telemedicine and embedded systems. Dr. Saini holds membership of many professional bodies. He is a life member of the IETE.

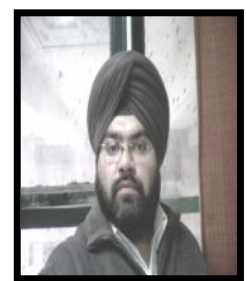

Amritpal Singh has completed his B.E. in Electrical and Electronics Engineering in 2002 and M.Tech in Instrumentation and Control Engineering in 2004.He is currently pursuing $\mathrm{PhD}$ from National Institute of Technology, Jalandhar.

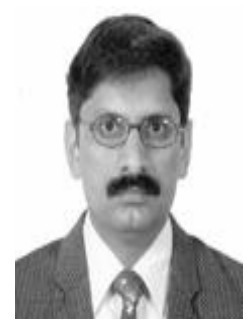

Dilbag Singh was born in Adyana (Panipat), on February 26, 1969. He received the B.E. (Hons.) degree in Electrical Engg from Punjab Engineering College, Chandigarh, and M.E. degree in Control \& Guidance from the University of Roorkee, and the Ph.D. degree in Engineering from the Indian Institute of Technology Roorkee in 1991, 1993, and 2004 respectively. The Ph.D. degree thesis was developed at the Instrumentation \& Signal Processing Laboratory of the Electrical Engineering Department under the direction of Vinod Kumar, S. C. Saxena and K. K. Deepak (AIIMS). After a brief stint at Goodyear India Limited, Faridabad, in September 1994 he joined the Department of Instrumentation and Control Engineering at of the Dr. B. R. Ambedkar National Institute of Technology, Jalandhar, and since 2005 has been Associate Professor of Instrumentation \& Control Engineering there, where he is teaching UG/PG courses related to biomedical signal processing and instrumentation. His professional research interests are in signal processing, in particular applied to biomedical applications. 